\title{
¿MACULOPATÍA MIÓPICA TRACCIONAL?
}

\section{MYOPIC TRACTION MACULOPATHY?}

\author{
FERNÁNDEZ-VEGA A ${ }^{1}$
}

El desarrollo de la tomografía de coherencia óptica (OCT) en los últimos años ha supuesto un tremendo avance en la exploración del polo posterior, hasta el punto de convertirse en una de las herramientas más útiles en la clínica diaria del especialista en retina. Es importante no solamente para el diagnóstico, o como herramienta de investigación para el conocimiento de la anatomía retiniana, sino que no se concibe hoy en día el abordaje quirúrgico de una gran parte de la patología retiniana, sin tener un estudio previo mediante OCT.

En los últimos tiempos, la OCT nos ha servido además para descubrir la existencia de toda una serie de enfermedades que, con el nivel de resolución de la biomicroscopía, no podíamos más que sospechar, abriendo una nueva vía para el posible tratamiento quirúrgico mediante vitrectomía, de estas afecciones. Una de estas enfermedades es la maculopatía miópica traccional (MMT).

Muchos pacientes miopes magnos que presentaban disminución visual central, sin neovascularización coroidea y sin otra causa que la justificara, eran «diagnosticados» de coroidosis miópica idiopática. Hoy sabemos que muchas de estas disminuciones visuales corresponden a esta patología macular, en muchos casos traccional, que tiene una alta prevalencia (1).

Tacano (2) en 1999 hace una primera descripción de la retinosquisis y el desprendimiento de retina foveal, como una patología frecuente en los miopes magnos con estafiloma posterior. Panozzo (3) utiliza por primera vez el término maculopatía miópica traccional (MMT), para referirse a estas patologías y realiza un estudio retrospectivo sobre 218 ojos en los que encontró tracción epiretiniana en el $46 \%$ y daño retiniano en el 34\%. Encontró que la patología macular traccional del miope, particularmente con estafiloma, comprendía las siguientes enfermedades, por orden de frecuencia:

Retinosquisis macular (otros autores hablan de foveosquisis).

Engrosamiento retiniano macular.

Agujero macular lamelar.

Desprendimiento de retina macular sin agujero macular (otros autores hablan de desprendimiento foveal).

El término MMT no ha cuajado entre los investigadores japoneses, autores de la mayoría de las publicaciones recientes sobre el tema, siendo por el
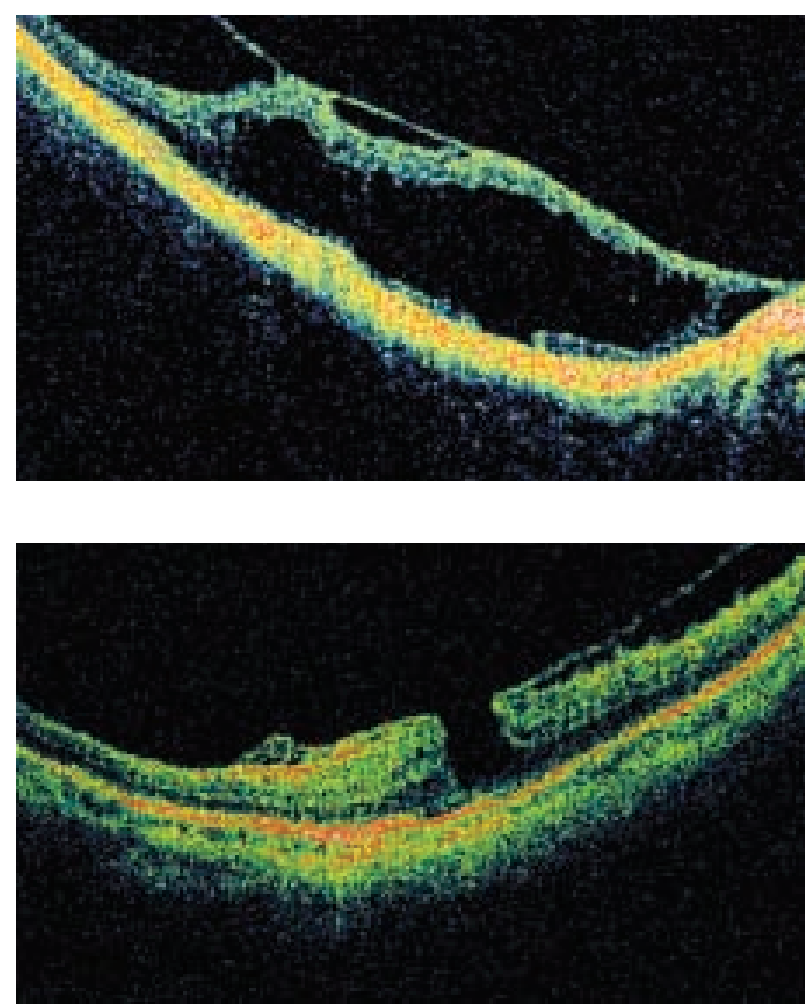

1 Doctor en Medicina. Oviedo. España.

E-mail: avega@cyberastur.es 


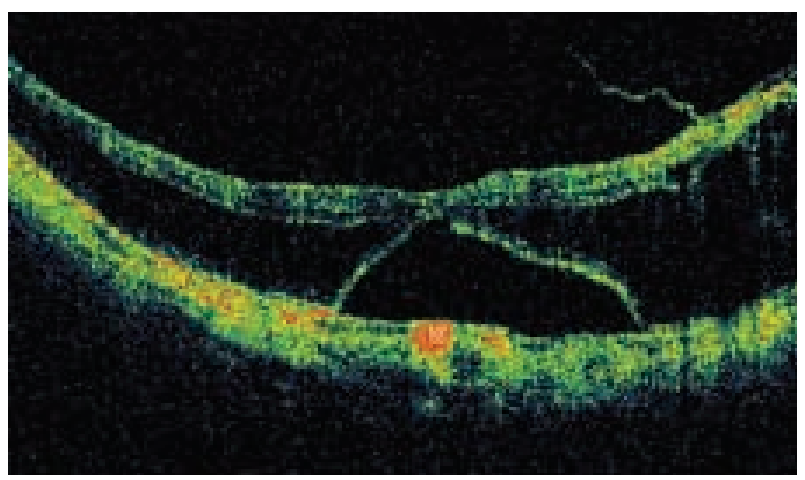

contrario muy utilizado en nuestro país entre los especialistas en retina. El posible motivo es que encontramos algunas de estas patologías sin que podamos constatar la presencia visible de tracciones maculares; es decir, podemos encontrar retinosquisis, foveosquisis o desprendimientos foveales, sin tracción vitreo-retiniana aparente. De esta manera, los últimos trabajos hay que buscarlos en la literatura bajo el nombre de retinosquisis, foveosquisis o desprendimientos foveales sin que hayan sido englobados bajo el término MMT.

Como posible explicación de los casos no traccionales, Tano et al $(4,5)$ han publicado recientemente la presencia de micropliegues retinianos que hacen relieve en la retina, en pacientes operados mediante vitrectomía por foveosquisis. Dichos micropliegues, que corresponderían a las arteriolas retinianas, estarían generados por la insuficiente elasticidad de las arteriolas retinianas durante la elongación del estafiloma del miope, al aumentar la longitud axial y ser las arteriolas rígidas. Tano concluye que las fuerzas que se ocasionan con el crecimiento del globo ocular y la retina, frente a las poco flexibles arteriolas, serían las responsables de determinadas patologías específicas de la miopía, como por ejemplo la foveosquisis miópica o los agujeros retinianos paravasculares.

Los primeros trabajos (6) consideraban que las patologías comprendidas en el término MMT, eran patologías muy estables. Sabemos que en muchas ocasiones son asintomáticas o que producen una sintomatología muy leve. Sin embargo últimamente algunos autores (7) consideran que su benignidad pueda ser solo aparente mostrándose como una enfermedad progresiva, con complicaciones frecuentes. Tanto unos como otros, concluyen que las complicaciones más severas en la evolución de la retinosquisis (el agujero macular y el desprendimiento de retina por agujero macular) están claramente relacionados con la tracción vítreo-macular.

Cualquier cirujano de vítreo-retina acostumbrado a operar en mácula del miope, sabe que no es una cirugía fácil ni mucho menos. La hialoides no sale en bloque, se rasga, hay que quitarla muchas veces con pinza, y las adherencias patológicas de la misma en el polo posterior, son tremendas. Estas adherencias, sobre todo a nivel de los vasos sanguíneos, son tan fuertes, que los desgarros iatrogénicos son muy frecuentes y en muchas ocasiones aunque hayamos liberado las tracciones del polo posterior, tenemos que finalizar la cirugía sin haber hecho una completa vitrectomía, con el riesgo que esto conlleva. Si además estudiamos las publicaciones hasta el momento sobre retinosquisis, foveosquisis, desprendimiento foveal y desprendimiento de retina sin agujero macular, el número de complicaciones es relativamente elevado, y las técnicas, diversas, si bien se pueden conseguir mejorías visuales (8-11).

¿Deben ser operados estos pacientes? Aún no tenemos la respuesta a esta pregunta. Como veíamos al principio, algunos autores piensan que la retinosquisis es estable, mientras que el trabajo de Shimada que presenta a la retinosquisis como una enfermedad progresiva está sustentado en el seguimiento de un número muy escaso de ojos (8 ojos).
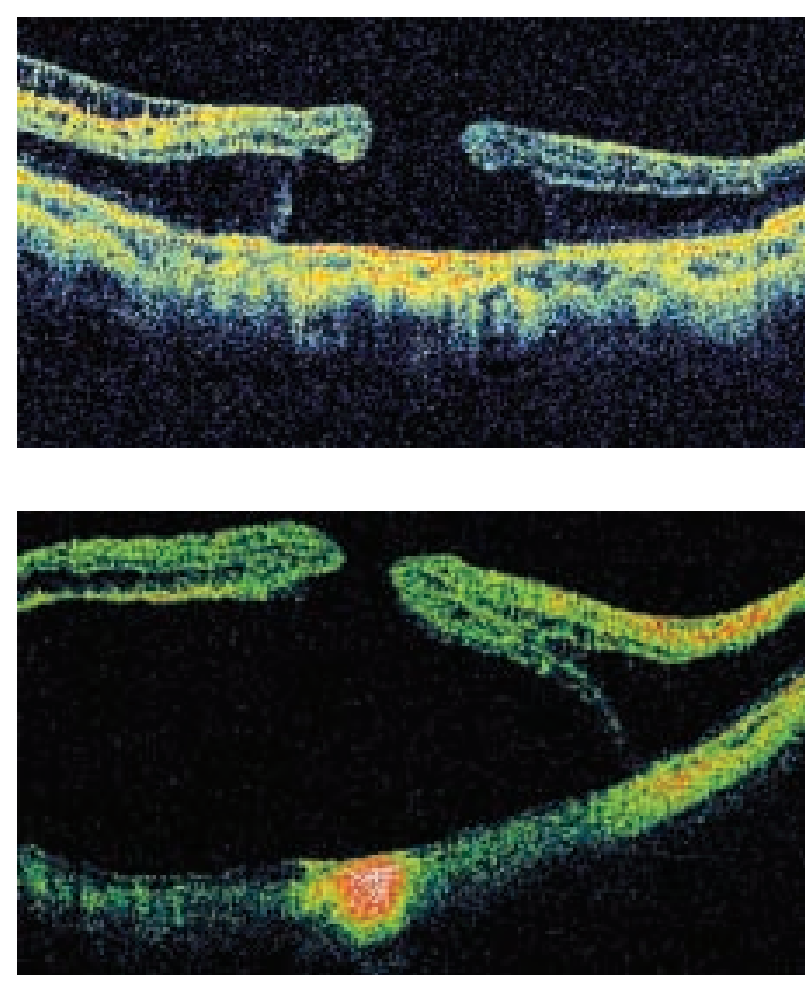
Es fundamental conocer la historia natural de la enfermedad y ser cautos a la hora de plantear una cirugía, pese a lo tentador que pueda ser cuando vemos la clara tracción a nivel de la mácula o cuando revisamos las últimas publicaciones, de expertos cirujanos japoneses, sobre el tratamiento de esta patología.

Probablemente acaben siendo quirúrgicos los casos de disminución progresiva de la visión y por el contrario los casos estables deberán ser simplemente observados.

Es muy probable que las patogenias de las enfermedades maculares antedichas y otras, como el agujero macular verdadero del miope magno, el desprendimiento de la retina por agujero macular y los desprendimientos de retina por agujeros lineales paravasculares o en polo posterior, estén íntimamente relacionadas entre sí.

Será nuestro trabajo en los próximos tiempos averiguar de que manera están relacionadas unas con otras e ir desentrañando la causa de las mismas y dilucidar la parte que corresponde en esta patogenia a la tracción vítreo-retiniana, la parte que corresponde a las fuerzas intrínsecas a la propia retina por inextensibilidad de su vascularización, o por las fuerzas que ocurren en los bordes de los estafilomas.

Creo que de cualquier manera, el término maculopatía miópica traccional no debe abandonarse, pues englobaría todas estas patologías en las que la tracción bien vítreo-retiniana, bien intrínseca, juega un papel fundamental.

Además de la apasionante aventura de encontrarnos ante una patología de reciente diagnóstico con todas las perspectivas que abre en el ámbito de la investigación, nos encontramos así mismo ante la posibilidad de tratamiento quirúrgico de una serie de enfermedades que hasta el momento eran causa de pérdida de agudeza visual de origen desconocido, en el paciente miope magno.

\section{BIBLIOGRAFÍA}

1. Baba T, Ohno-Matsui K, Futagami S, Yoshida T, Yasuzumi K, Kojima A, Et al. Prevalence and characteristics of foveal retinal detachment without macular hole in high myopia. Am J Ophthalmol 2003; 135: 338-342.

2. Takano M, Kishi S. Foveal retinoschisis and retinal detachment in severely myopic eyes with posterior staphiloma. Am J Ophthalmol 1999; 128: 472-476.

3. Panozzo G, Mercanti A. Optical coherence tomography findings in myopic traction maculopathy. Arch Ophthalmol 2004; 122: 1455-1460.

4. Ikuno Y, Gomi F, Tano Y. Potent retinal arteriolar traction as a possible cause of myopic foveoschisis. Am J Ophthalmol 2005; 139: 462-467.

5. Sayanagi K, Ikuno Y, Gomi F, Tano Y. Retinal vascular microfolds in highly myopic eyes. Am J Ophthalmol 2005, 139: 658-663.

6. Benhamou N, Massin P, Haouchine B, Erginay A, Gaudric A. Macular retinoschisis in highly myopic eyes. Am J Ophthalmol 2002; 133: 794-800.

7. Shimada N, Ohno-Matsui K, Baba T, Futagami S, Tokoro T, Mochizuki M. Natural course of macular retinoschisis in highly myopic eyes without macular hole or retinal detachment. Am J Ophthalmol 2006; 142: 497-500.

8. Hirakata A, Hida T. Vitrectomy for myopic posterior retinoschisis or foveal detachment. Jpn J Ophthalmol 2006; 50: 53-61.

9. Kobayashi H, Kishi S. Vitreous surgery for highly myopic eyes with foveal detachment and retinoschisis. Ophthalmology 2003; 110: 1702-1707.

10. Baba T, Tanaka S, Maesawa A, Teramatsu T, Noda Y, Yamamoto S. Scleral buckling with macular plombe for eyes with myopic macular retinoschisis and retinal detachment without macular hole. Am J Ophthalmol 2006; 142: 483-487.

11. Sayangi K, Ikuno $Y$, Tano $Y$. Reoperation for persistent myopic foveoschisis after primary vitrectomy. Am J Ophthalmol 2006; 141: 414-417. 